Chirurg 2014 $\cdot 85: 723$

DOI 10.1007/s00104-014-2843-6

Online publiziert: 25 . Juli 2014

(c) Springer-Verlag Berlin Heidelberg 2014

O. Strobel · M.W. Büchler

Klinik für Allgemein-, Viszeral- und Transplantationschirurgie, Universität Heidelberg

\title{
Notwendigkeit einer strukturierten Nachbeobachtung nach Pankreasresektion bei intraduktal papillär muzinöser Neoplasie
}

siegrades, des IPMN-Typs (MD, BD) und des histologischen Subtyps analysiert.

\section{Ergebnisse}

Von den 366 Patienten hatten 82 niedriggradige Dysplasien, 171 mittelgradige Dysplasien, 45 hochgradige Dysplasien und 68 eine invasive IPMN. Während einer mittleren Nachbeobachtungszeit von 44,4 Monaten traten bei $39(10,7 \%)$ Patienten Rezidive auf (im Median nach 18,3 Monaten). 12 Patienten (3,3\%) hatten eine erneute IPMN im Restpankreas, $4(1,1 \%)$ entwickelten ein Pankreaskarzinom, 9 (2,5\%) hatten ein lokoregionäres Rezidiv am Ansetzungsrand und 24 Patienten (6,6\%) entwickelten ein systemisches Rezidiv. Von diesen 39 Patienten wurden 6 (15\%) reoperiert, 28 (72\%) chemotherapiert und 5 (13\%) weiter beobachtet. 9 Patienten verstarben am Rezidiv. Der Dysplasiegrad war stark mit dem Rezidivrisiko assoziiert: $33,8 \%$ bei invasivem IPMN, 13,3\% bei hochgradigen und $4 \%$ bei niedrig- bis mittelgradigen Dysplasien $(\mathrm{p}<0,001)$. Bei MD/MT-IPMN war das Rezidivrisiko signifikant höher als beim BD-IPMN (15,3 vs. 7,7\%; p=0,021). Die Rezidivhäufigkeit war signifikant mit dem histologischen Subtyp assoziiert: gastrisch $4,7 \%$, intestinal $12,5 \%$, pankreatobiliär $30,2 \%$, onkozytisch $0 \%(\mathrm{p}<0,001)$. In der multivariaten Analyse waren ein erhöhtes präoperatives Carbohydrate-Antigen 19-9, hochgradige Dysplasien und eine invasive Komponente unabhängig mit dem Rezidivrisiko assoziiert. Das Vorliegen einer nichtinvasiven Komponente am Absetzungsrand war nicht mit einem erhöhten Rezidivrisiko verbunden. Das 5-Jahres-Gesamtüberleben betrug 86,6\% und war signifikant mit dem Dysplasiegrad assoziiert (invasive IPMN: 72,2\%, hochgradig: $83,2 \%$, niedrig- bis mittelgradig: 90,2\%; $\mathrm{p}=0,003$ ).

\section{Diskussion}

Die Autoren folgern, dass nach Resektion eines IPMN eine sorgfältige postoperative Nachsorge empfohlen werden muss und zwar nicht nur für Patienten mit invasivem IPMN, sondern auch für Patienten mit prämalignen IPMNs, insbesondere wenn hochgradige Dysplasien vorliegen. Das Auftreten systemischer Rezidive bei 6 von 298 (2,0\%) als nichtinvasiv diagnostizierten IPMNs deutet auf die Gefahr falsch-negativer Diagnosen mit Übersehen invasiver Anteile hin.

Die Autoren empfehlen eine Nachsorge für mindestens 5 Jahren, wobei diese zeitliche Empfehlung nicht durch weitere Daten untermauert wird.

Diese Studie ist wichtig, weil sie die Notwendigkeit einer strukturierten Nachsorge nach Resektion einer IPMN unterstreicht und dabei zum ersten Mal auf die Bedeutung von Dysplasie, IPMN-Typ und histologischem Subtyp für das Rezidivrisiko eingeht.

\section{Korrespondenzadresse}

PD Dr. O. Strobel

Klinik für Allgemein-, Viszeralund Transplantationschirurgie, Universität Heidelberg, Im Neuenheimer Feld 110, 69120 Heidelberg Oliver.Strobel@med.uni-heidelberg.de

Interessenkonflikt. O. Strobel und M.W. Büchler geben an, dass kein Interessenkonflikt besteht. erweitert. Die Rezidivhäufigkeiten wurden insbesondere im Kontext des Dyspla- 